\title{
Linear Polarization of Lunar Emission
}

\section{R. D. Davies and F. F. Gardner}

\section{CSIRO, Sydney, Australia}

Using the Australian CSIRO 210-ft telescope at wavelengths of $6 \mathrm{~cm}, 11.3 \mathrm{~cm}$, and $21 \mathrm{~cm}$, measurements were made of the linear polarization of the emission from the moon. The observed values of the polarized intensity at different distances from the center of the moon were compared with theoretical curves calculated by taking the values for a smooth dielectric sphere and convolving them with the aerial polar diagram. The observations fit the curves quite well if values of $\epsilon=2.0,2.1$, and 2.3 are used at wavelengths of $6 \mathrm{~cm}, 11.3 \mathrm{~cm}$, and $21 \mathrm{~cm}$, respectively. These values of the dielectric constant are much lower than the values inferred from radar observations $(\sim 2.7)$. Similar results have been reported by other observers.

It has been suggested that if allowance were made for the effects of surface roughness this discrepancy might be removed. However, Krotikov and Troitsky [Uspekii, 81, 589, 1963] have investigated theoretically the effects of roughness, and show that it should broaden the observed curves, particularly on the side toward the center of the disk. As there is no evidence for this broadening in the experimental data, it seems that roughness cannot explain the discrepancy.

(Paper 69D12-605) 